\title{
Dank an die Gutachter des Jahres 2013
}

Bei der Begutachtung und Durchsicht von RöFo-Manuskripten haben uns im Jahr 2013 zahlreiche Wissenschaftler in Kliniken und Instituten unterstützt, die nachfolgend genannt sind und denen wir - die Herausgeber und Mitwirkenden der RöFo - für Ihre Hilfe herzlich danken. Zunächst genannt seien die Gutachter, die für ihr besonderes Engagement ausgezeichnet wurden:

Robert Ritzel (Hamburg)

Martin Fiebich (Gießen)

Christian Habermann (Hamburg)

G. Adam, Hamburg

R. Adamus, Nürnberg

T. Albrecht, Berlin

H. Alfke, Lüdenscheid

S. Alibek, Erlangen

H. Alkadhi, Zürich

C. Althoff, Berlin

H. Amthauer, Magdeburg

A. Artmann, Wels

P. Baltzer, Jena

A. Ba-Ssalamah, Wien

T. Bäuerle, Hamburg

H. Bauknecht, Berlin

F. Baum, Göttingen

M. Beer, Graz

P. Begemann, Düsseldorf

K. Beiderwellen, Essen

M. Bendszus, Würzburg

T. Bernhardt, Hannover

L. Berthold, Hannover

D. Beyersdorff, Berlin

J. Biederer, Heidelberg

H.-J. Biersack, bonn

C. Binkert, Winterthur

P. Blanke, Freiburg

C. Blendl, Köln

T. Bley, Hamburg

W. Block, Bonn

D. Blondin, Düsseldorf

H. Bolte, Kiel

M. Born, Bonn

H. Bovenschulte, Köln

P. Brader, Wien

R. Braunschweig, Halle

D. Briem, Hamburg

P. Bruners, Aachen

R. Bucek, Wien

C. Buchbender, Düsseldorf

A. Bücker, Homburg/Saar

B. Buerke, Münster

M. Burg, Münster

H.-P. Busch, Trier

M. Cejna, Feldkirch

A. Chavan, Oldenburg

A. Chemelli, Innsbruck

S. Clasen, Tübingen

C. Claussen, Tübingen
E. Coppenrath, München

C. Czerny, Wien

F. Dammann, Göppingen

M. Das, Aachen

S. Delorme, Heidelberg

T. Denecke, Berlin

M. Deppe, Münster

T. Derlin, Hamburg

K. Detmar, Nürnberg

M. Dewey, Berlin

S. Diederich, Düsseldorf

R. Dorffner, Eisenstadt

T. Durmus, Berlin

M. Düx, Frankfurt am Main

R. Eibel, Schwerin

T. Engelhorn, Erlangen

R. Erbel, Essen

U. Ernemann, Tübingen

B. Ertl-Wagner, München

K. Ewen, Duisburg

P. Eysel, Köln

E. Fallenberg, Berlin

J. Fiebach, Berlin

M. Fiebich, Giessen

B. Fiedler, Münster

J. Fiehler, Hamburg

R. Fischbach, Hamburg

T. Fischer, Berlin

U. Fischer, Göttingen

L. Fischer, Hamburg

T. Flohr, Forchheim

E. Foert, Berlin

M. Forsting, Essen

T. Franiel, Jena

B. Frericks, Berlin

M. Freund, Aschaffenburg

M. Fuchsjaeger, Wien

D. Führer, Essen

J. Fütterer, Nijmegen

S. Garbe, Bonn

B. Gebauer, Berlin

D. Geisel, Berlin

E. Gizewski, Innsbruck

C. Glüer, Kiel

S. Goericke, Essen

D. Görlich, Münster

D. Gosch, Leipzig
A. Gossmann, Köln

F. Götz, Hannover

A. Grams, Innsbruck

A. Graser, München

J. Grimm, New York

R. Grimmer, Erlangen

W. Gross-Fengels, Hamburg

U. Grzyska, Hamburg

C. Guhl, Nürnberg

R. Günther, Aachen

Ch. R. Habermann, Hamburg

S. Hähnel, Heidelberg

P. Hallscheidt, Heidelberg

R. Hammerstingl, Frankfurt am Main

E. Hattingen, Frankfurt

K. Hausegger, Klagenfurt

S. Heiland, Heidelberg

W. Heindel, Münster

T. Helmberger, München

H. Henkes, Stuttgart

K. Hering, Dortmund

J. Herrmann, Hamburg

K. Herz, Tübingen

V. Heßelmann, Hamburg

P. Heusch, Düsseldorf

L. Heuser, Bochum

T. Heusner, Hamm

J. Heverhagen, Bern

S. Heywang-Köbrunner, München

I. Hilger, Jena

W. Hirsch, Leipzig

R.-T. Hoffmann, Dresden

E. Hofmann, Fulda

A. Hojreh, Wien

N. Hosten, Greifswald

A. Huppertz, Berlin

H. Imhof, Wien

A. Jödicke, Berlin

K. Jungnickel, Magdeburg

M. Kachelriess, Heidelberg

A. Kaim, Aarau

B. Kammer, München

C. Kampmann, Mainz

M. Katoh, Krefeld

M. Kaul, Hamburg

T. Kemper, Dornstadt

J. Kettenbach, Bern 
D. Kienzl, Wien

C. Kinstner, Wien

J. Kirchner, Hagen

D. Klee, Duesseldorf

J. Klisch, Erfurt

U. Klose, Tübingen

S. Kloska, Erlangen

T. Kluba, Tübingen

m. koehler, Münster

F. Koerber, Köln

M. Köhler, Münster

A. Koops, Hamburg

S. Kösling, Halle

H. Köstler, Würzburg

N. Kraemer, Aachen

K.-F. Kreitner, Mainz

B. Kress, Frankfurt

C. Krestan, Wien

S. Kreuzer, Wien

G. Krombach, Gießen

K. Krug, Köln

R. Kubik-Huch, Baden

M. Kuefner, Erlangen

H. Kugel, Münster

C. Kuhl, Aachen

H. Kühl, Essen

C. Kulinna-Cosentini, Wien

G. Kurlemann, Münster

J. Lammer, Wien

M. Laniado, Dresden

R. Lanzman, Düsseldorf

W. Lehmann, Hamburg

A. Lemke, Bremen

H. Lenzen, Münster

S. Leschka, St.Gallen

S. Ley, München

J. Ley-Zaporozhan, Heidelberg

T. Liebig, Köln

T. Linden, Münster

J. Linn, München

C. Loewe, Wien

R. Loose, Nürnberg

J. Lotz, Göttingen

P. Lunkenheimer, Augsburg

H. Madjar, Wiesbaden

A. Mahnken, Marburg

A. Maier, Wien

D. Maintz, Münster

T. Mang, Wien

R. Matthias, Heidelberg

W. Matzek, Wien

M. Maurer, Berlin

M. Mayerhoefer, Wien

M. Memarsadeghi, Wien

B. Meyer, Hannover

H. Michaely, Mannheim

P. Mildenberger, Mainz

W. Moeller-Hartmann, Meppen

A. Mohr, Göttingen

N. Morakkabati-Spitz, Bonn

G. Mostbeck, Wien
W. Mueller-Forell, Mainz

U. Mueller-Lisse, Muenchen

A. Mundinger, Osnabrück

C. Naehle, Bonn

J. Nagarajah, Essen

S. Niehues, Berlin

V. Niem, Stuttgart

K. Nikolaou, München

C. Nimsky, Marburg

C. Nolte-Ernsting, Mülheim an der Ruhr

S. Obenauer, Düsseldorf

J. Oestmann, Berlin

H. Omran, Münster

C. Ozdoba, Bern

K. Peldschus, Hamburg

B. Pfleiderer, Münster

M. Pitton, Mainz

P. Pokieser, Wien

H. Portugaller, Graz

V. Prasad, Berlin

D. Prayer, Wien

M. Puderbach, Heidelberg

R. Puls, Erfurt

A. Quante, Berlin

H. Quick, Erlangen

H.-J. Raatschen, Hannover

T. Rand, Wien

M. Regier, Hamburg

W. Reith, Homburg/Saar

B. Renger, München

F. Rengier, Heidelberg

A. Ringelstein, Essen

R. Ritzel, Hamburg

T. Roeren, Aarau

P. Rogalla, Berlin

J. Rothe, Berlin

R. Röttgen, Berlin

D. Rüfenacht, Zürich

E. Rummeny, München

J. Sandstede, Hamburg

A.-O. Schaefer, Freiburg

C. Schaefer-Prokop, Amsterdam

C. Schäffeler, München

M. Scharitzer, Wien

R. Schernthaner, Wien

C. Scheurig-Muenkler, Berlin

F. Schick, Tübingen

H. Schild, Bonn

M. Schlamann, Essen

L. Schmidt, Münster

R. Schmidt, Münster

C. Schmidt, Münster

B. Schmitz, Ulm

O. Schober, Münster

A. Schober, Münster

M. Schoder, Wien

S. Schrading, Aachen

L. Schreiber, Mainz

A. Schreyer, Regensburg

C. Schüller-Weidekamm, Wien

D. Schulze, Freiburg
M. Schwaiger, München

B. Schweiger, Essen

W. Schwindt, Münster

H. Seifarth, Münster

S. Singh, Boston

p. skaane, Oslo

A. Sommer, Münster

I. Sorge, Leipzig

G. Staatz, Mainz

G. Stamm, Hannover

M. Steinborn, München

W. Steinbrich, Basel

M. Stenzel, Jena

C. Stippich, Basel

C. Stroszczynski, Regensburg

T. Struffert, Erlangen

C. Stueckle MD, Castrop-Rauxel

J. Tacke, Passau

C. Taschner, Freiburg

K. Tesdal, Friedrichshafen

D. Thomas, Bonn

C. Thomas, Tübingen

H. Thraen, Berlin

M. Töpker, Wien

B. Turowski, Düsseldorf

M. Uder, Erlangen

M. Uffmann, Neunkirchen

M. Uhl, Freiburg

L. Umutlu, Essen

T. Vogl, Frankfurt

H. von Boetticher, Bremen

C. von Falck, Hannover

T. von Kalle, Stuttgart

D. Vorwerk, Ingolstadt

F. Wacker, Hannover

H.-J. Wagner, Berlin

M. Walz, Eschborn

I. Wanke, Essen

M. Warmuth-Metz, Würzburg

C. Weber, Hamburg

S. Weigel, Münster

A. Wenke, Münster

H. Wersching, Münster

J. Weßling, Münster

J. Westhof, Kassel

T. Westhoff, Berlin

A. Wetter, Essen

E. Wiener, Berlin

M. Wiesmann, Aachen

R. Wiewrodt, Münster

K. Wilhelm, Bonn

W. Willinek, Bonn

S. Wirth, München

K.-J. Wolf, Berlin

F. Wolf, Wien

D. Wormanns, Berlin

K. Wörtler, München

S. Zangos, Frankfurt 